\title{
Microprocessor dynamics shows co- and post-transcriptional processing of pri-miRNAs
}

\author{
ANNITA LOULOUPI, EVGENIA NTINI, JULIA LIZ, and ULF ANDERSSON ØROM \\ Max Planck Institute for Molecular Genetics, 14195 Berlin, Germany
}

\begin{abstract}
miRNAs are small regulatory RNAs involved in the regulation of translation of target transcripts. miRNA biogenesis is a multistep process starting with the cleavage of the primary miRNA transcript in the nucleus by the Microprocessor complex. Endogenous processing of pri-miRNAs is challenging to study and the in vivo kinetics of this process is not known. Here, we present a method for determining the processing kinetics of pri-miRNAs within intact cells over time, using a pulse-chase approach to label transcribed RNA during $15 \mathrm{~min}$, and follow the processing within a 1-hour window after labeling with bromouridine. We show that pri-miRNAs exhibit different processing kinetics ranging from fast over intermediate to slow processing, and we provide evidence that pri-miRNA processing can occur both cotranscriptionally and post-transcriptionally.
\end{abstract}

Keywords: miRNA; miRNA biogenesis; pri-miRNA

\section{INTRODUCTION}

MicroRNAs (miRNA) are small RNAs that mediate posttranscriptional regulation of gene expression (Kim et al. 2009). miRNAs are transcribed as primary transcripts as long as $30 \mathrm{~kb}$ and processed by the Microprocessor complex in the nucleus (Gregory et al. 2004). The Microprocessor complex is the minimal complex required for pri-miRNA processing in vitro, consisting of the two proteins, Drosha and DGCR8 (Gregory et al. 2004).

Several features, such as sequence motifs around the precursor miRNA (pre-miRNA) hairpin in the primary miRNA (pri-miRNA) transcript (Auyeung et al. 2013; Conrad et al. 2014) and within the hairpin loop (Auyeung et al. 2013), have been shown to be involved in processing efficiency in mammals. Furthermore, early studies of primiRNA transcripts have reported a cotranscriptional processing by the Microprocessor complex (Kim and Kim 2007; Morlando et al. 2008), using mostly in vitro assays and studies of single endogenous examples. The retention of pri-miRNAs at chromatin by factors such as HP1BP3 has also been suggested to be important for efficient pri-miRNA processing (Liu et al. 2016). The state-of-the-art methodologies are, however, not able to address the general and in vivo dynamics of pri-miRNA processing.

We have previously shown that sequencing of the chromatin-associated RNA can reveal the steady-state processing efficiency of individual pri-miRNAs within the cell, demon-

Corresponding author: oerom@molgen.mpg.de

Article is online at http://www.rnajournal.org/cgi/doi/10.1261/rna 060715.117. Freely available online through the RNA Open Access option. strating pri-miRNA processing as one of the most important factors for determining the level of mature miRNAs (Conrad et al. 2014). To further follow processing of pri-miRNAs endogenously over time without constraints of their cellular localization or differential transcription, we pulse-labeled RNA with bromouridine $(\mathrm{BrU})$ and followed its processing during a 1-h chase (Paulsen et al. 2013). We show that primiRNAs exhibit different processing kinetics both within the same polycistronic transcript and with respect to transcription and release from chromatin.

\section{RESULTS}

\section{Setup of RNA pulse-chase sequencing}

RNA sequencing yields an average view of RNA in the cell or in the respective purified subcellular compartment, reflecting a mixture of RNA of different age compared to the time of transcription. To follow RNA from transcription through processing, actively transcribed RNA can be labeled with a pulse of a modified nucleotide (e.g., BrU) (Paulsen et al. 2013 ) that allows for subsequent purification of RNA of a defined age during chase after stopping the labeling (Fig. 1A).

We have previously shown that RNA sequencing of chromatin-associated RNA to a very high depth reveals a processing signature around the location precursor miRNAs (pre-miRNA) that reflects the processing efficiency of

(C) 2017 Louloupi et al. This article, published in RNA, is available under a Creative Commons License (Attribution-NonCommercial 4.0 International), as described at http://creativecommons.org/licenses/by-nc/4.0/. 

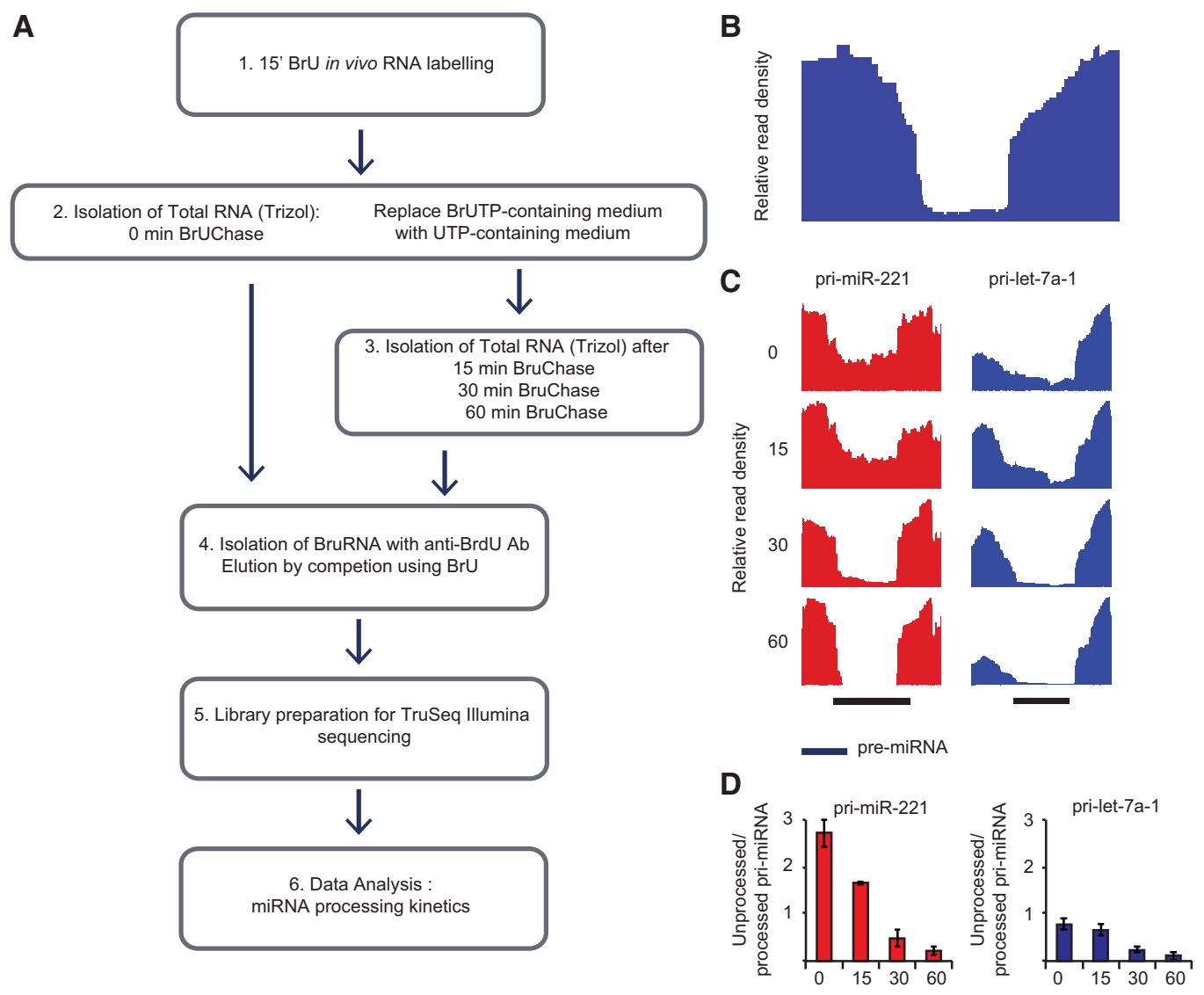

FIGURE 1. Measuring pri-miRNA processing kinetics with nascent RNA labeling. ( $A$ ) Workflow for RNA pulse labeling with BrU and chase to follow nascent RNA. (B) Concept of processing signature in pri-miRNAs. Processing extent is calculated as the read density in the pre-miRNA region compared to the flanking regions. Processing efficiency is calculated as $(1-$ processing extent). (C) Processing signatures in RNA-sequencing data from nascent RNA in pulse-chase experiment for pri-miR-221 and pri-let-7a-1. (D) Quantification by PCR of unprocessed/processed pri-miRNA, for example, shown in $C$ from two independent experiments. The $y$-axes show the relative read density as a window of minimum-to-maximum read counts within the window.

individual pri-miRNAs by the Microprocessor complex (Fig. 1B; Conrad et al. 2014). While this approach provides insight into the average processing efficiency of steady-state primiRNA associated to chromatin, it cannot address the dynamics of this process. To expand our previous studies of steady-state pri-miRNA processing efficiency, we pulselabeled RNA during transcription by adding BrU to the medium for a short time (15 min) and subsequently chased for $0,15,30$, and $60 \mathrm{~min}$ after $\mathrm{BrU}$ was removed from the medium to follow the kinetics of the processing (Fig. 1A). We subjected total BrU-labeled RNA from all time points obtained from HEK293 cells to next-generation sequencing using an Illumina Hi-Seq 2500 to obtain $\sim 200 \mathrm{M}$ reads per sample, and we analyzed pri-miRNA processing at each time point for the transcripts shown in Supplemental Table 1. Processing efficiency was calculated by extracting the read counts covering the miRNA and the summed read counts from the two surrounding 100-nt intervals upstream of and downstream from the $5^{\prime}$ and $3^{\prime}$ ends of the miRNA, respectively, after extending it by $20 \mathrm{nt}$ on both ends to avoid the region where coverage of RNA-sequencing reads might be affected by the proximity to the end generated by the cleavage by the Microprocessor complex, as described in Materials and Methods. We restricted the further analysis to 38 pri-miRNAs, where we see high coverage of the pri-miRNA transcript and a pronounced processing profile (Supplemental Table 1).

\section{In vivo profiles of processing kinetics from whole cells}

After pulse labeling of RNA, we follow the processing of primiRNAs during a 60-min time-course (chase) in HEK293 cells. Interestingly, for the 38 pri-miRNAs where we see a pronounced profile, we observed differences in the processing kinetics across pri-miRNA transcripts and within polycistronic pri-miRNAs. We categorized these 38 pri-miRNAs into three groups according to the extent of the processing profile during the chase: Fast processed; Intermediate processed; and Slow processed. The profiles for miR-221 and let-7a- 1 are shown in Figure 1C, representing an intermediate processed $\left(t_{1 / 2} \sim 40 \mathrm{~min}\right)$ and a fast processed $\left(t_{1 / 2}<15 \mathrm{~min}\right)$ pri-miRNA, respectively. These processing efficiencies can be recapitulated using quantitative PCR of individual pri- 

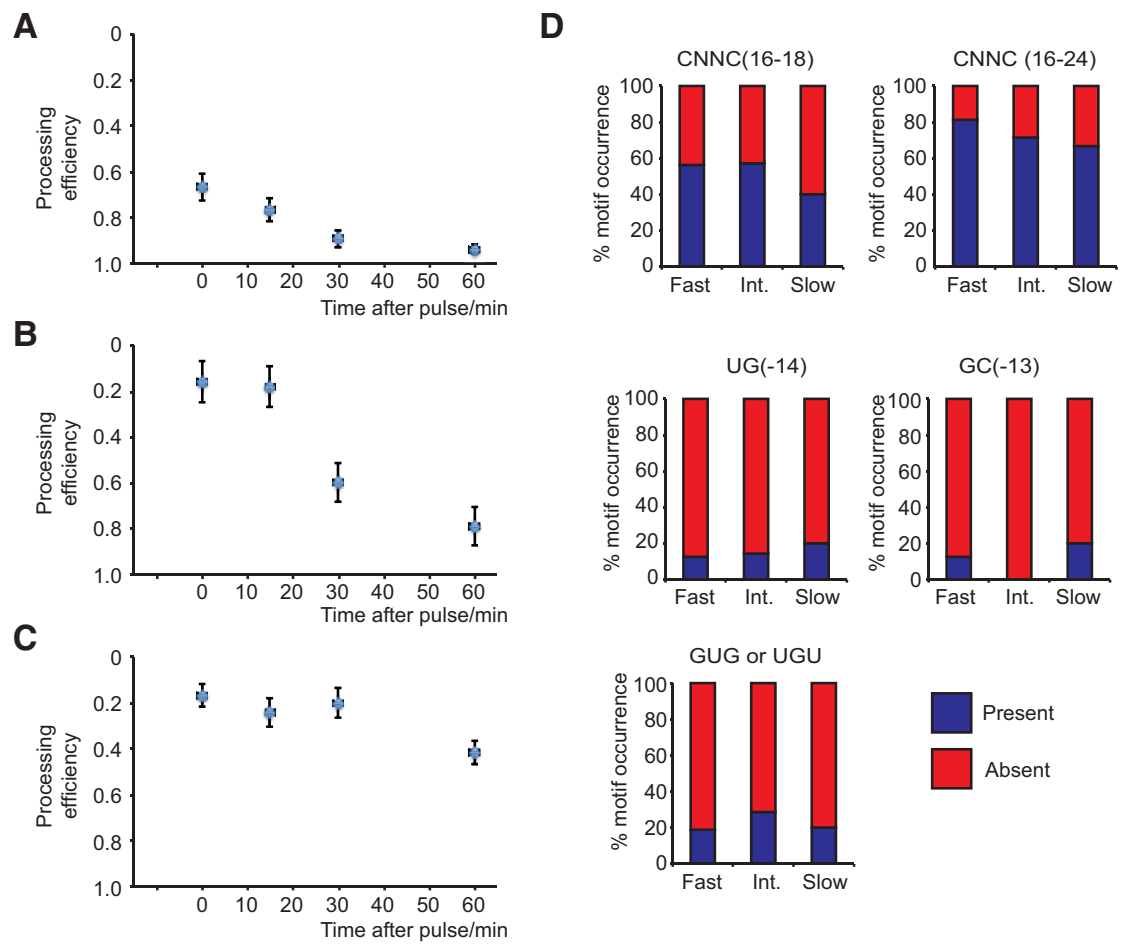

FIGURE 2. Pri-miRNA processing kinetics and associated motifs. Average processing profile for (A) Fast processed pri-miRNAs $(n=16),(B)$ Intermediate processed pri-miRNAs $(n=7)$, and $(C)$ Slow processed pri-miRNAs $(n=15)$. $(D)$ Motif occurrence for known motifs associated with pri-miRNA processing efficiency depicted as relative occurrence for each of the groups of pri-miRNA processing kinetics.

miRNAs. Using primers spanning the processing site we can determine the relative amounts of unprocessed pri-miRNAs, and taking the ratio to the level of total pri-miRNA (processed + unprocessed) we can estimate the processing efficiency for single pri-miRNA transcripts, as described in Conrad et al. (2014). Representative results from the PCR quantification of pri-miRNA processing for miR-221 and let-7a-1 are shown in Figure 1D.

\section{Pri-miRNAs show differential kinetics in processing}

Our data demonstrate that some pri-miRNAs show almost complete processing at time point $0 \mathrm{~h}$, while others exhibit a slower pattern of processing by the Microprocessor (Supplemental Table 1). For several pri-miRNAs, we observe a very low processing efficiency at $0^{\prime}$ (after $15^{\prime} \mathrm{BrU}$ labeling, 0 -min chase), arguing that we are able to capture some pri-miRNAs even before the processing by the Microprocessor complex takes place. The average processing efficiencies of Fast processed (Fig. 2A), Intermediate processed (Fig. 2B), and Slow processed (Fig. 2C) are shown. For Fast processed pri-miRNAs we observe the majority of Microprocessor activity within the pulse (before $0^{\prime}$ chase), while for Intermediate processed pri-miRNAs the majority of processing happens around $15^{\prime}-30^{\prime}$ of chase.
For the group of Slow processed primiRNAs, most processing occurs after $30^{\prime}$. While the fast and the intermediate processed pri-miRNAs all obtain a complete processing within $60^{\prime}$, several of the slow processed pri-miRNAs are $<50 \%$ processed at the $60^{\prime}$ time point.

We assessed whether there is a correlation between the expression level of pri-miRNAs and the processing kinetics (Supplemental Fig. 1) based on chromatin-associated RNA-sequencing data from Conrad et al. (2014). The median expression level for the three groups are comparable, albeit higher than the average expression of pri-miRNAs in HEK293 cells, reflecting that the sequencing-based assay captures the highly expressed pri-miRNAs with highest confidence. In agreement with this, we have previously reported that the processing efficiency of pri-miRNAs in steady-state chromatin-associated RNA is not dependent on the expression level of the primiRNA. In addition, the levels of mature miRNAs, as determined by small RNA sequencing (Conrad et al. 2014), for fast and intermediate processed pri-miRNAs are similar on average, whereas the expression of mature miRNAs from slow processed pri-miRNAs is lower on average, with an expression distribution similar to all expressed miRNAs in HEK293 cells (Supplemental Fig. 2).

The presence of sequence motifs around the pre-miRNA and within the hairpin loop have been shown to affect the processing efficiency of pri-miRNAs (Auyeung et al. 2013; Conrad et al. 2014), namely the UG(-14) 14 nt upstream of the pre-miRNA (Auyeung et al. 2013); GC(-13) $13 \mathrm{nt}$ upstream of the pre-miRNA (Conrad et al. 2014); the CNNC motif 16-18 (Auyeung et al. 2013; Roden et al. 2017) or 16-24 (Conrad et al. 2014) nt downstream from the premiRNA as well as the GUG or UGU stem-loop motif (Auyeung et al. 2013). While these motifs are reported to associate with better processing only the CNNC motif is abundantly present in mammalian pri-miRNAs (Auyeung et al. 2013). We see the CNNC motif in more than half of the pri-miRNAs analyzed with a slight preference for Fast over Intermediate over Slow processed pri-miRNAs, especially when considering the CNNC(16-24) motif (Fig. 2D), that is, however, not statistically significant for any of the three groups of pri-miRNAs. This would be in agreement with CNNC being a general motif involved in pri-miRNA processing and not as a binding site for cofactors regulating the kinetics of processing. We do not see a general enrichment of the UG(-14), GU(-13), or the GUG or UGU motifs for the analyzed pri-miRNAs (Fig. 2D). 
A

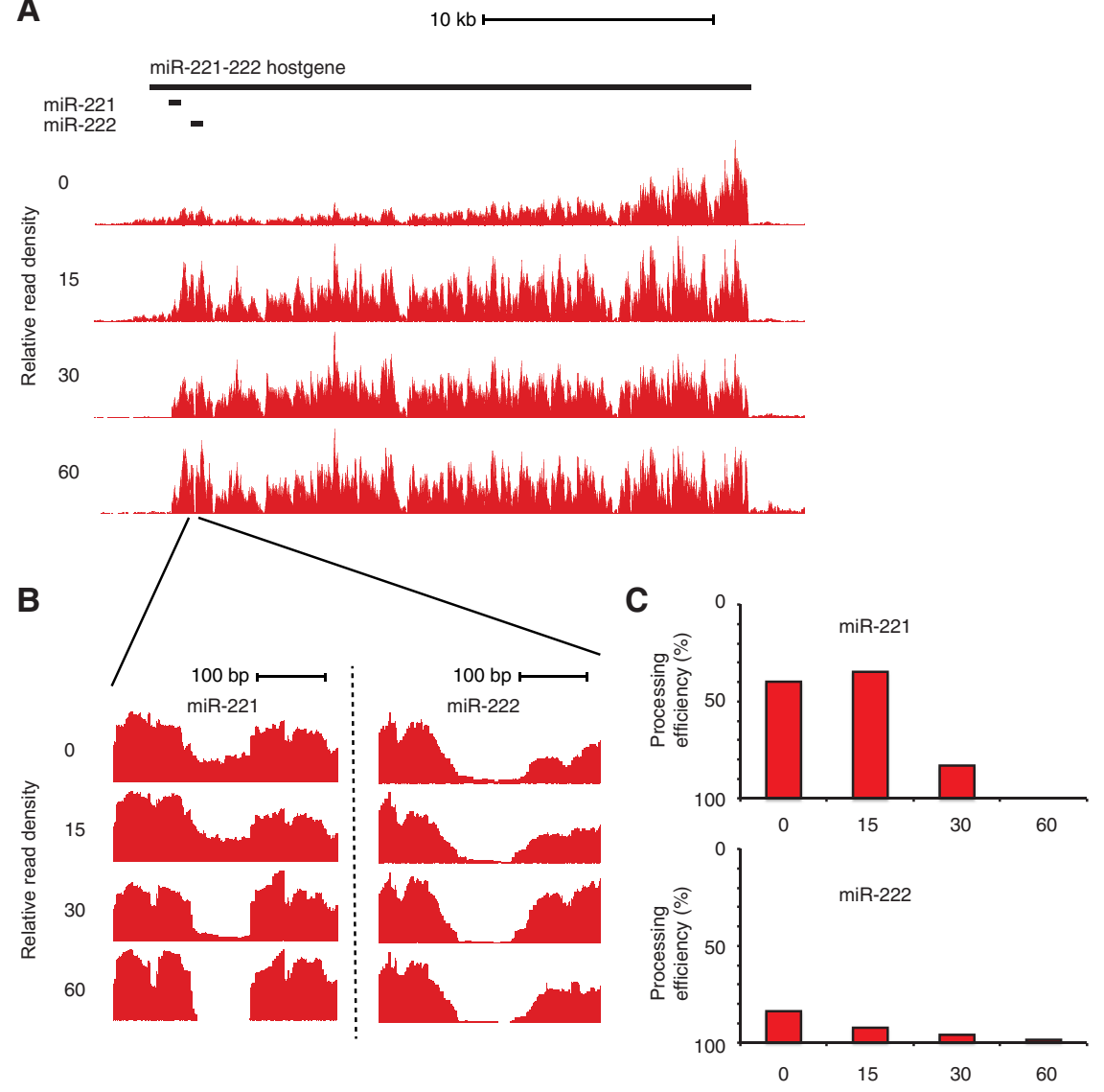

FIGURE 3. Differential processing within the pri-miR-221/222 polycistronic pri-miRNA transcript. (A) Overview of the genomic region and full pri-miRNA transcript. (B) Enlarged read densities around pre-miRNAs for miR-221 and miR-222. (C) Quantification of processing efficiency from $B$.

\section{Differential processing within polycistronic pri-miRNAs}

Several miRNAs are expressed from polycistronic primiRNAs (Du et al. 2015), and these miRNAs often belong to the same families and thus are predicted to target the same mRNAs for translation regulation and RNA degradation (Eichhorn et al. 2014). Prominent polycistronic primiRNAs are let-7a/f and miR-221/222, described to have important roles in cancer and cell cycle (le Sage et al. 2007). We observe differential processing kinetics within both these polycistronic pri-miRNAs. The miR-221/222 pri-miRNA is a 25-kb-long transcript (Fig. 3A) encoding two miRNAs. While the two miRNAs are relatively closely spaced, they exhibit very different processing kinetics (Fig. 3B,C), demonstrating that processing kinetics are not defined by the primary transcript or its association to chromatin, as has recently been suggested (Liu et al. 2016). miR-221 and miR-222 are both shown to affect the cell cycle by targeting the p27 tumor suppressor and to promote cancer progression (le Sage et al. 2007). The processing efficiency of miR-221 and miR-222 over time was quantified as described in Figure 1B and shown in Figure 3C. Interestingly, the expression levels of miR-221 and miR-222 in HEK293 cells are comparable and the half-life of the mature miRNAs have been estimated in mouse to be the same (Marzi et al. 2016), suggesting that processing kinetics could define the biological regulation modulating the levels of miRNAs against a specific target throughout the cell cycle.

\section{Pri-miRNAs are processed co- and post-transcriptionally}

To estimate the release from chromatin of pri-miRNAs, we assumed that the processing efficiency as derived from steady-state chromatin-associated primiRNA data (Conrad et al. 2014) reflects the average processing efficiency of primiRNAs while associated to chromatin. The duration of association to chromatin of an individual pri-miRNA prior to release can then be estimated by the dynamics of processing efficiency after pulse labeling of RNA and chase. The average age of individual pri-miRNAs at chromatin, and thereby their time of release, is thus reflected by the corresponding processing efficiency and can be used to estimate at what time transcripts are released from chromatin within each of the three groups of pri-miRNAs. Using the processing efficiency at chromatin derived from steady-state chromatin-associated RNA-sequencing data (Conrad et al. 2014) and comparing it to processing efficiency for individual pri-miRNAs during the chase, we determine the release of pri-miRNA transcripts from chromatin (Fig. 4A-C, shaded area). The Fast processed pri-miRNAs (Fig. 4A) show very high processing efficiency when quantified from chromatin-associated RNA (Conrad et al. 2014). While we estimate the chromatin-release of Fast processed pri-miRNAs to occur 7-14 min after chase, the processing half-life is around $8 \mathrm{~min}$ and is reached within the pulse (Fig. 4A, dashed lines), arguing that these transcripts are truly cotranscriptionally processed and never leave chromatin. For the group of Intermediate processed primiRNAs, we estimate chromatin-release to 19-33 min after pulse and the processing half-life to $41 \mathrm{~min}$ (26 min after the chase) (Fig. 4B, dashed lines). This suggests that while cotranscriptional processing of this group of pri-miRNAs is generally inefficient, the kinetics increase at the release of the pri-miRNA transcript from chromatin, or when the transcript is loosely associated to chromatin. The Very slow processed pri-miRNAs show little processing within the first 

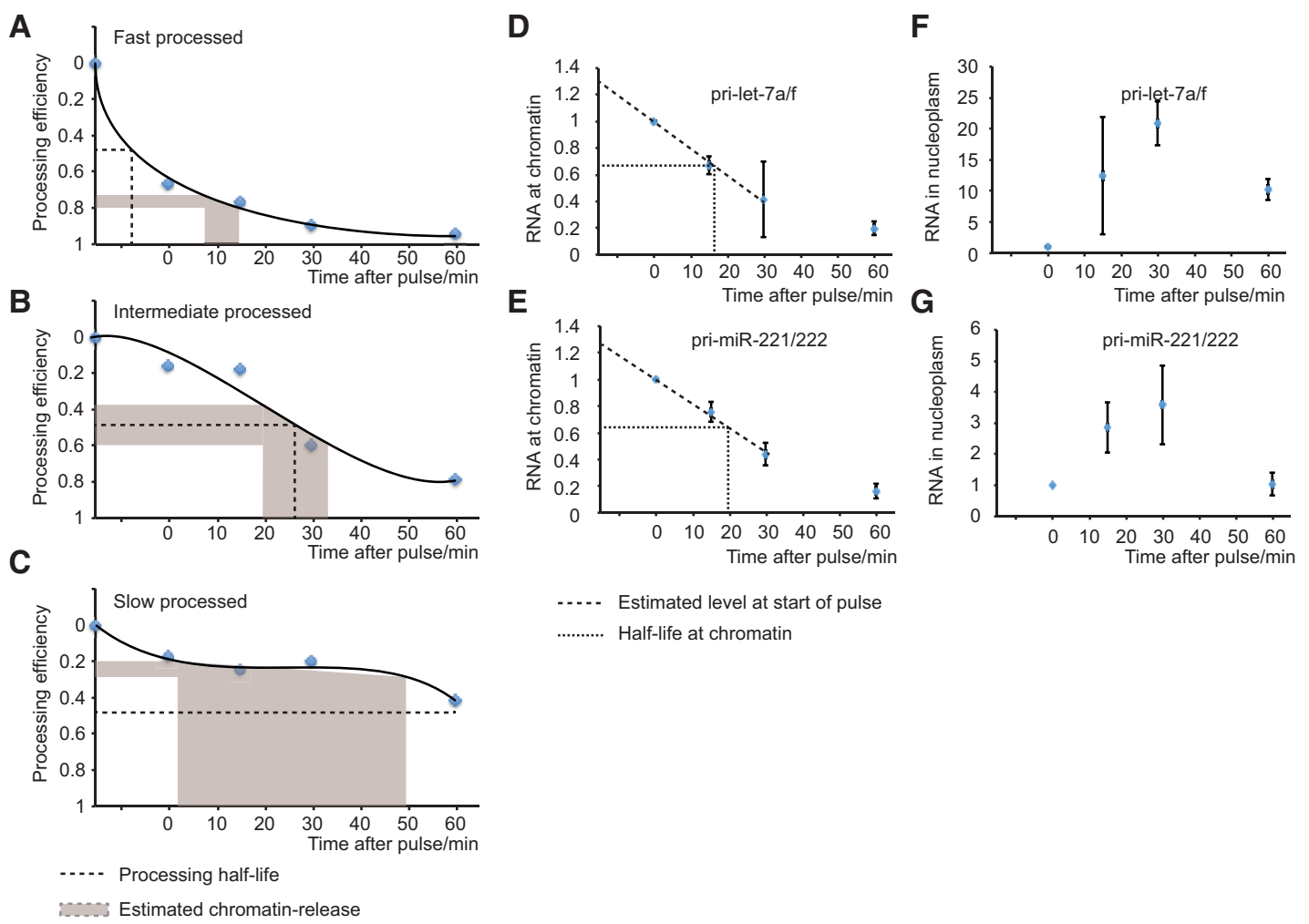

.... Half-life at chromatin

FIGURE 4. Chromatin-associated pri-miRNA processing and release. Chromatin-release (shaded area) and processing half-life (dashed lines) are determined for $(A)$ Fast, $(B)$ Intermediate, and $(C)$ Slow processed pri-miRNAs. $(D, E)$ Experimental validation of the release from chromatin of $(D)$ pri-let-7a/f and $(E)$ pri-miRNA-221/222. Dashed lines show estimated pri-miRNA at time $0^{\prime}$ (time of BrU addition), and fine dashed lines show estimated half-life at chromatin. $(F, G)$ Relative amount of pri-miRNA in the nucleoplasm normalized to $15^{\prime}$. Experiments in $D-G$ are from three independent experiments.

$60 \mathrm{~min}$ and do not reach $50 \%$ on average within the $60 \mathrm{~min}$ applied chase in this study (Fig. 4C).

To support this approach of determining chromatin-release, we isolated the chromatin and nucleoplasmic fractions of cells after pulse labeling with BrU and chase for the same time points (Fig. 4D-G). Here, we show that for both miR$221 / 222$ and let-7a/f, the half-life at chromatin is $17-19$ min, in agreement with our estimate from the dynamic primiRNA processing efficiency and in support of our proposed model where Intermediate processed pri-miRNAs are more efficiently processed post-transcriptionally at their release from chromatin (Fig. 4D,E). We furthermore show that pri-miRNAs accumulate in the nucleoplasmic fraction until $30^{\prime}$ after the BrU-pulse, supporting an incomplete processing at chromatin (Fig. 4F,G).

\section{DISCUSSION}

While previous work has proposed pri-miRNA processing to be an exclusively cotranscriptional event (Kim and Kim 2007; Morlando et al. 2008), it also suggested Drosha cleavage of the pri-miRNA as a relatively slow process (Morlando et al. 2008). To this end, we observe processing half-lives of the groups of pri-miRNAs of 8 and $41 \mathrm{~min}$, respectively, for the Fast and Intermediate processed pri-miRNAs. This is not in agreement with an exclusively cotranscriptional processing but fits well with Drosha cleavage being a relatively slow process. We observe that the group of Intermediate processed pri-miRNAs is most efficiently processed at the time of release from chromatin or at a stage coinciding with the transcript half-life at chromatin where the transcript is being released from chromatin. This stage happens after the transcript has been completely transcribed, and we therefore call it post-transcriptional processing. This could be a state where the RNA becomes more available to catalytic nucleoplasmic proteins or less available to inhibitory proteins tightly bound to chromatin.

While factors responsible for chromatin retention (Liu et al. 2016) or chromatin release could play an important role in this process, we see chromatin release of pri-miRNAs at comparable times after transcription for pri-miRNA transcripts showing different processing kinetics, arguing that chromatin retention is not the determining factor for pri-miRNA processing kinetics. In fact, the Fast processed pri-miRNAs show a slightly faster release from chromatin than the Intermediate and Slow processed pri-miRNAs, in contrast to a model where a tighter association to chromatin increases processing efficiency, as suggested in Liu et al. (2016). 
Drosha, the active part of the Microprocessor complex in cleaving pri-miRNAs, has been shown to be recruited to pri-miRNAs at chromatin cotranscriptionally (Morlando et al. 2008), in some cases by the RNA-binding protein FUS (Morlando et al. 2012). A possible scenario explaining that processing does not take place immediately for Intermediate and Slow processed pri-miRNAs could be that inhibitory factors associating to chromatin prevent cotranscriptional processing. As Drosha is recruited cotranscriptionally, these proteins could inhibit the activity of the chromatin-associated Microprocessor complex. Dissociation from chromatin would then lead to less interaction with these inhibitory factors and more efficient processing of the primiRNA transcripts. Identification of such factors would reveal important novel insights into the chromatin-RNA interactions and dynamics responsible for proper pri-miRNA processing and is an interesting challenge for future work.

\section{MATERIALS AND METHODS}

\section{Tissue culture and labeling of RNA}

HEK293 cells were cultured in DMEM growth medium supplemented with $10 \%$ fetal bovine serum (FBS) under normal growth conditions $\left(37^{\circ} \mathrm{C}\right.$ and $\left.5 \% \mathrm{CO}_{2}\right)$. The day before labeling with $\mathrm{BrU}$, $\sim 5.0 \times 10^{6}$ cells were seeded in 150 -mm plates. Cells were $70 \%-$ $80 \%$ confluent before the addition of $\mathrm{BrU}$. BrU (-5-Bromouridine cat. no. CAS 957-75-5 Santa Cruz Biotechnology) was added to a final concentration of $2 \mathrm{mM}$ to the media and cells were incubated at normal growth conditions for $15 \mathrm{~min}$ (pulse). Cells were washed three times in PBS and either collected directly (0-h chase time point) or chased in conditional media supplemented with $20 \mathrm{mM}$ uridine (Sigma cat. no U3750-25G) for 15, 30, or $60 \mathrm{~min}$. RNA was purified using TRIzol following manufacturer's instructions.

\section{Preparation of labeled RNA}

Labeled RNA was isolated as described in Paulsen et al. (2013) with some modifications. Of note, $35 \mu \mathrm{L}$ of anti-mouse IgG magnetic Dynabeads (Invitrogen) were transferred to a $1.5 \mathrm{~mL}$ microfuge Protein Low binding tube and washed three times with $1 \times \mathrm{BrU}-\mathrm{IP}$ buffer $(0.1 \%$ BSA in RNAse-free PBS). After the final wash, the beads were resuspended with $1 \times$ BrU-IP buffer supplemented with SUPERase In RNase Inhibitor 1:2000 together with BrdU antibody ( $5 \mu \mathrm{g}$ of antibody per $100 \mu \mathrm{g}$ RNA). Antibody-beads mixture was incubated for $1 \mathrm{~h}$ at RT with gentle rotation followed by three washes with $1 \times \mathrm{BrU}$-IP buffer. Of note, $150 \mu \mathrm{g}$ RNA was used for each BrU-IP and heated for $5 \mathrm{~min}$ at $65^{\circ} \mathrm{C}$ prior to IP; $5 \times \mathrm{BrU}$-IP buffer $(0.5 \%$ BSA $5 \times$ PBS supplemented with SUPERase In RNase Inhibitor 1:2000) was added to the RNA to obtain a final concentration of $1 \times$. RNA-antibody-beads mixture was incubated for $60 \mathrm{~min}$ at room temperature with gentle rotation in a final volume of $0.8 \mathrm{~mL}$. The beads were washed three times with $800 \mu \mathrm{L} 1 \times \mathrm{BrU}$ IP at RT, rotating for $5 \mathrm{~min}$ each wash. For elution, $200 \mu \mathrm{L}$ of Elution buffer $(0.1 \%$ BSA and $25 \mathrm{mM}$ bromouridine in PBS) was added directly on the beads and the tubes were incubated for $1 \mathrm{~h}$ with continuous shaking $(1100 \mathrm{rpm})$ at $4^{\circ} \mathrm{C}$. The supernatant (elu- ate) was transferred to a new tube and RNA was precipitated by adding one-tenth volumes of $3 \mathrm{M}$ sodium acetate $(\mathrm{pH} 5.2)$ and 3 volumes of pure ethanol. RNA was precipitated at $-80^{\circ} \mathrm{C}$ overnight. RNA pellet was washed twice with $75 \%$ ethanol and resuspended in RNase-free water. RNA quality was analyzed using Agilent 2100 Bioanalyzer with an Agilent RNA 6000 Pico kit according to the manufacturer's instructions. For the chromatin-release assay, cells were labeled with $\mathrm{BrU}$ and chased as described above. After the chase for the respective time points, cells were fractionated as described in Conrad and Ørom (2017) on ice to obtain the chromatin pellet from which RNA was extracted. BrU-labeled RNA was then isolated as described above. Analysis using qPCR was done using the same number of cells for each condition.

\section{Quantitative real-time PCR}

RNA was reverse transcribed using the Goscript reverse transcription Promega A500 cDNA kit and quantified on a 7900HT Fast real-time PCR system (Applied Biosystems) using the Go Taq qPCR Master Mix Promega (A6001).

\section{Primer sequences}

pri-let7a1/d/f1 FW GCATTTGTTTATGGCCTGGA pri-let7al/d/f1 RV CACCCCCATCCAGTGTACTT pri-let7a1 unprocessed FW ACACCCACCACTGGGAGATA let7al unprocessed RV GCCTGGATGCAGACTTTTCT pri-221/2 FW AGCAAAGAGAACACCAATCCTGT pri-221/2 RV GTTCCAAGCTTTCCTCCCATGAT pri-mir221 unprocessed FW ACTTGCAAGCTGAACATCCA pri-mir221 unprocessed RV TGCCTAACGAACACAGAAATCT

\section{RNA sequencing and data analysis}

Library preparation was performed using the Trueseq Stranded Total RNA Kit (Illumina). Sequencing was performed on an Illumina HiSeq 2500 instrument to obtain around $200 \mathrm{M}$ reads per sample. Reads were mapped to GRCh37 (hg19) using STAR version 2.4.2.a (Dobin et al. 2013) with default parameters. To assess the processing efficiency, we extracted the read counts covering the miRNA and the summed read counts from two surrounding 100-nt intervals upstream of and downstream from the $5^{\prime}$ and $3^{\prime}$ end of the miRNA, respectively, after extending it by $20 \mathrm{nt}$ on both ends. The processing efficiency is the ratio of the miRNA read counts to the surrounding read counts, multiplied by the ratio 200 to the miRNA length.

\section{Filtering and annotation of miRNAs}

MicroRNAs used in the analysis were filtered to include only highconfidence microRNAs showing the absence of other noncoding RNA species in the region; folding of the pre-miRNA into a hairpin; and homogenous reads in small-RNA-sequencing data for both the $5^{\prime}$ and $3^{\prime}$ mature miRNA. We required conservation of the hairpin structure in orthologous members of the gene family for conserved microRNAs (as defined in mirBase) including mouse or other mammals and conservation of the seed in more than $50 \%$ of the orthologous genes. The miRNAs used are from Conrad et al. 
(2014) and include 229 miRNAs: 138 classified as broadly conserved; 52 classified as weakly conserved; and 39 as nonconserved. We determined the exact Microprocessor cleavage sites using the annotation of the $5 \mathrm{p}$ and $3 \mathrm{p}$ miRNA strands from miRBase and mapped them onto the sequence of the pre-miRNA.

\section{DATA DEPOSITION}

Data from RNA sequencing are deposited in GEO with accession number GSE92565. The small RNA sequencing data are available from GEO with accession number GSE56862.

\section{SUPPLEMENTAL MATERIAL}

Supplemental material is available for this article.

\section{ACKNOWLEDGMENTS}

E.N. has been funded by a postdoctoral stipend from the Alexander von Humboldt Foundation. Work in the author's laboratory is funded by the German Research Council (DFG) and the Alexander von Humboldt Foundation through the Sofja Kovalevskaja Award to U.A.Ø.

Author contributions: A.L. did experimental work, interpreted data, conceived experiments, and wrote the paper. E.N. did computational analysis, interpreted data, and wrote the paper. J.L. did experimental work, conceived experiments, and interpreted data. U.A.Ø. conceived the experiments, interpreted data, supervised research, and wrote the paper. All authors read and approved the paper.

Received January 9, 2017; accepted February 21, 2017.

\section{REFERENCES}

Auyeung VC, Ulitsky I, McGeary SE, Bartel DP. 2013. Beyond secondary structure: primary-sequence determinants license pri-miRNA hairpins for processing. Cell 152: 844-858.

Conrad T, Ørom UA. 2017. Cellular fractionation and isolation of chromatin-associated RNA. Methods Mol Biol 1468: 1-9.
Conrad T, Marsico A, Gehre M, Orom UA. 2014. Microprocessor activity controls differential miRNA biogenesis in vivo. Cell Rep 9: $542-554$.

Dobin A, Davis CA, Schlesinger F, Drenkow J, Zaleski C, Jha S, Batut P, Chaisson M, Gingeras TR. 2013. STAR: ultrafast universal RNA-seq aligner. Bioinformatics 29: 15-21.

Du P, Wang L, Sliz P, Gregory RI. 2015. A biogenesis step upstream of microprocessor controls miR-17 92 expression. Cell 162: 885-899.

Eichhorn SW, Guo HL, McGeary SE, Rodriguez-Mias RA, Shin C, Baek D, Hsu SH, Ghoshal K, Villén J, Bartel DP. 2014. mRNA destabilization is the dominant effect of mammalian microRNAs by the time substantial repression ensues. Mol Cell 56: 104-115.

Gregory RI, Yan KP, Amuthan G, Chendrimada T, Doratotaj B, Cooch N, Shiekhattar R. 2004. The Microprocessor complex mediates the genesis of microRNAs. Nature 432: 235-240.

Kim YK, Kim VN. 2007. Processing of intronic microRNAs. EMBO J 26: 775-783.

Kim VN, Han J, Siomi MC. 2009. Biogenesis of small RNAs in animals. Nat Rev Mol Cell Biol 10: 126-139.

le Sage C, Nagel R, Egan DA, Schrier M, Mesman E, Mangiola A, Anile C, Maira G, Mercatelli N, Ciafrè SA, et al. 2007. Regulation of the $\mathrm{p} 27^{\mathrm{Kip} 1}$ tumor suppressor by miR-221 and miR-222 promotes cancer cell proliferation. EMBO J 26: 3699-3708.

Liu HM, Liang CY, Kollipara RK, Matsui M, Ke X, Jeong BC, Wang ZQ, Yoo KS, Yadav GP, Kinch LN, et al. 2016. HP1BP3, a chromatin retention factor for co-transcriptional microRNA processing. Mol Cell 63: $420-432$.

Marzi MJ, Ghini F, Cerruti B, de Pretis S, Bonetti P, Giacomelli C, Gorski MM, Kress T, Pelizzola M, Muller H, et al. 2016. Degradation dynamics of microRNAs revealed by a novel pulsechase approach. Genome Res 26: 554-565.

Morlando M, Ballarino M, Gromak N, Pagano F, Bozzoni I, Proudfoot NJ. 2008. Primary microRNA transcripts are processed co-transcriptionally. Nat Struct Mol Biol 15: 902-909.

Morlando M, Dini Modigliani S, Torrelli G, Rosa A, Di Carlo V, Caffarelli E, Bozzoni I. 2012. FUS stimulates microRNA biogenesis by facilitating co-transcriptional Drosha recruitment. EMBO J 31: 4502-4510.

Paulsen MT, Veloso A, Prasad J, Bedi K, Ljungman EA, Tsan YC, Chang CW, Tarrier B, Washburn JG, Lyons R, et al. 2013. Coordinated regulation of synthesis and stability of RNA during the acute TNF-induced proinflammatory response. Proc Natl Acad Sci 110: 2240-2245.

Roden CA, Gaillard J, Kanoria S, Rennie W, Barish S, Cheng J, Pan W, Liu J, Cotsapas C, Ding Y, et al. 2017. Novel determinants of mammalian primary microRNA processing revealed by systematic evaluation of hairpin-containing transcripts and human genetic variation. Genome Res 27: 374-384. 

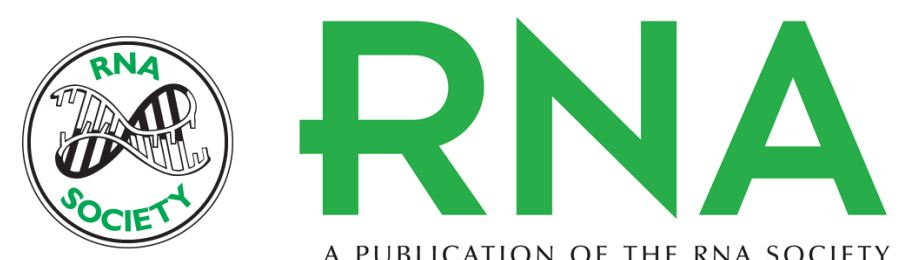

A PUBLICATION OF THE RNA SOCIETY

\section{Microprocessor dynamics shows co- and post-transcriptional processing of pri-miRNAs}

Annita Louloupi, Evgenia Ntini, Julia Liz, et al.

RNA 2017 23: 892-898 originally published online March 1, 2017

Access the most recent version at doi:10.1261/rna.060715.117

\section{Supplemental http://rnajournal.cshlp.org/content/suppl/2017/03/01/rna.060715.117.DC1 Material}

References This article cites 16 articles, 6 of which can be accessed free at: http://rnajournal.cshlp.org/content/23/6/892.full.html\#ref-list-1

Open Access Freely available online through the RNA Open Access option.

Creative This article, published in RNA, is available under a Creative Commons License Commons (Attribution-NonCommercial 4.0 International), as described at License http://creativecommons.org/licenses/by-nc/4.0/.

Email Alerting Receive free email alerts when new articles cite this article - sign up in the box at the Service top right corner of the article or click here.

\section{|||||||| Providing Precise Solutions for your research.}

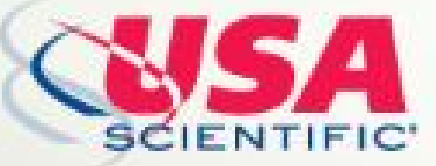

To subscribe to $R N A$ go to:

http://rnajournal.cshlp.org/subscriptions

(C) 2017 Louloupi et al.; Published by Cold Spring Harbor Laboratory Press for the RNA Society 